\title{
COMPARISON OF INTEGRAL EQUATION PREDICTIONS AND \\ EXPERIMENTAL BACKSCATTER MEASUREMENTS FROM RANDOM CONDUCTING ROUGH SURFACES
}

\author{
C. E. Nance, A. K. Fung, and J. W. Bredow \\ Wave Scattering Research Center \\ University of Texas at Arlington, UTA Box 19016 \\ Arlington, Texas, U.S.A. 76019
}

\section{ABSTRACT}

The scattering behavior from randomly rough surface targets has been analyzed by comparing the predictions of an integral equation model for surface scattering ( $\mathrm{Li}$ and Fung, PIERS' 89 , pg.144) to experimental data. This model has also been checked by numerical simulation (Chen and Chen, IGARSS' 89, pg.1300). Experimental data was taken for the purpose of the comparison of trends and levels of scattering strength versus frequency, incidence angle, and polarization. The surface parameters chosen will allow comparison in the low frequency region, the intermediate frequency region, and into the physical optics limit. Two of the targets were constructed by a numerically controlled mill to produce surfaces with predefined statistical characteristics. The third target is a two scale surface, constructed by hammering an aluminum plate. The statistics for this surface were compiled after the construction.

Key words: Scattering, Rough Surface, Backscatter

\section{Introduction}

The phenomenon of scattered waves from rough surfaces have been investigated from many viewpoints. Theoretical approaches have analyzed the processes through low- and high-frequency approximations. Two scale models followed in later years to attempt to combine the behavior of the two approximations to have a theory which would agree with measurements at small and large incidence angles. The behavior at the intermediate frequencies where neither of the frequency approximations were satisfied was still unclear. A scattering model for a perfectly conducting random surfaces was developed by Fung and Pan [1] to come up with a iterative approach for solving for the scatter fields. This method has been shown to match well with 2-D moment method solutions for low-, intermediate-, and high-frequency ranges [2]. This method was extended to finitely conducting random surfaces by
$\mathrm{Li}$ and Fung [3]. The physical generation of statistically known surface targets is time consuming and expensive, but the exact control over the roughness was required to be able to test the different frequency regions of the surface. The intermediatefrequency region is the region of interest because numerous measurements in the high- and low-frequency regions have been published in the past.

Section II will present a description of the theory used in the algorithm which produced the comparison curves for the experimental measurements. The rough surface target generation and description will follow in Section III. Section IV will present the measurement system and the data reduction techniques. The results and figure discussion will be given in Section V.

\section{Theoretical Descrintion}

The theoretical basis of the algorithm used in producing the predicted behavior for the scatter fields is based on the work by $\mathrm{Li}$ and Fung [3] for backscattering from finitely conducting rough surfaces. The expressions in this work have been shown to convert back to the correct expressions for the limiting case of a perfectly conducting rough surface. These general expressions also produce the Kirchhoff model predictions in the high frequency limit and the correct small perturbation model behavior in the low frequency limit.

This Integral Equation Method (IEM) by Li and Fung uses the approach of an analytic iteration for the surface currents, and then calculating the far-zone scattered field by integrating the surface current. This analytic iteration assumes that if the initial estimate for the surface currents is close, the first iteration will improve the surface current distribution to the correct values. The first guess used in the derivation is the Kirchhoff current distribution. The iteration produces an addition current term containing an integral including the Kirchhoff current distribution. The initial guess of the current distribution is associated with high frequency behavior, but with 
the added current term from the analytic iteration, the model produces predictions which agree with small perturbation models in low-frequency regions. The integral equation model also has been verified by numerical moment method solutions [2].

The evaluation of the analytic expressions produce a series solution which converges very quickly unless $k \sigma$ is very large. For this case a high frequency solution could be used for faster computation. For the case of Gaussian correlation function, the spectrum used in the series expressions is known, and the computation time is practically instantaneous on a computer with comparable speed of a VAX 8800.

The expressions for the like-polarized backscatter coefficients are given in EQ(1) and EQ(4).

$$
\begin{gathered}
\sigma_{\mathrm{vv}}^{\circ}=\frac{1}{2} k_{1}^{2}\left|f_{\mathrm{vv}}\right|^{2} e^{-4 \sigma^{2} k_{z}^{2}} \sum_{n=1}^{\infty} \frac{\left(4 \sigma^{2} k_{z}^{2}\right)^{n}}{n !} W^{(n)}\left(-2 k_{x}, 0\right) \\
+\frac{1}{8} k_{1}^{2}\left|F_{v v}\left(k_{x}, 0\right)\right|^{2} e^{-2 \sigma^{2} k_{z}^{2}} \sum_{n=1}^{\infty} \frac{\left(\sigma^{2} k_{z}^{2}\right)^{n}}{n !} W^{(n)}\left(-2 k_{x}, 0\right) \\
+\frac{1}{2} k_{1}^{2} \operatorname{Re}\left\{f_{v v}^{*} F_{v v}\left(k_{x}, 0\right) e^{-3 \sigma^{2} k_{z}^{2}}\right. \\
\left.* \sum_{n=1}^{\infty} \frac{\left(2 \sigma^{2} k_{z}^{2}\right)^{n}}{n !} W^{(n)}\left(-2 k_{x}, 0\right)\right\} \text { EQ(1) }
\end{gathered}
$$

Where

$$
\begin{aligned}
F_{v v}\left(k_{x}, 0\right) & =2\left[1+R_{\prime \prime}\right]^{2}\left[1-\frac{1}{\varepsilon_{\mathrm{r}}}\right] \frac{\sin ^{2} \theta}{\cos \theta} \\
\mathrm{f}_{\mathrm{v} v} & =\frac{2 \mathrm{R}_{\prime \prime}^{\prime \prime}}{\cos \theta}
\end{aligned}
$$

$$
\begin{aligned}
& \sigma_{h h}^{o}=\frac{1}{2} k_{1}^{2}\left|f_{h h}\right|^{2} e^{-4 \sigma^{2} k_{z}^{2}} \sum_{n=1}^{\infty} \frac{\left(4 \sigma^{2} k_{z}^{2}\right)^{n}}{n !} W^{(n)}\left(-2 k_{x}, 0\right) \\
& +\frac{1}{8} k_{1}^{2}\left|F_{h h}\left(-k_{x}, 0\right)\right|^{2} e^{-2 \sigma^{2} k_{z}^{2}} \sum_{n=1}^{\infty} \frac{\left(\sigma^{2} k_{z}^{2}\right)^{n}}{n !} W^{(n)}\left(-2 k_{x}, 0\right)
\end{aligned}
$$$$
+\frac{1}{2} k_{1}^{2} \operatorname{Re}\left\{f_{h h}^{*} F_{h h}\left(-k_{x}, 0\right) e^{-3 \sigma^{2} k_{z}^{2}}\right.
$$

$$
\left.* \sum_{n=1}^{\infty} \frac{\left(2 \sigma^{2} k_{2}^{2}\right)^{n}}{n !} W^{(n)}\left(-2 k_{x}, 0\right)\right\}
$$

Where

$$
\begin{gathered}
F_{h h}\left(-k_{x}, 0\right)=8 R_{\perp} \frac{\sin ^{2} \theta}{\cos \theta} \\
f_{h h}=-\frac{2 R_{\perp}}{\cos \theta}
\end{gathered}
$$

The quantity $\mathrm{W}^{(\mathrm{n})}(\mathrm{)})$, is the nth order power of the spectrum associated with the surface correlation function. These series solution expressions for the scattering coefficients are the reduced expressions from the general bistatic expressions. The integral equation model can also produce cross-polarized predictions, but at this time only the like polarized behavior will be compared to experimental measurements.

\section{Target Description}

Two of the rough surface targets measured were generated as coordinatre grids on a workstation computer and constructed using a numerically controlled mill. The generation of the surface grid coordinates is based on the work by $\mathrm{J}$. Rochier [4]. The third surface target was constructed by beating a piece of aluminum with three different sized ball-ping hammers. The two machined rough surface patches are circular targets, (1.2m diameter), with known surface height deviation and correlation lengths. The third hammered target has been analyzed to provide approximate values for the surface height variation and the correlation profile shape. The two machined surfaces were milled from four foot square structural foam sheets. The surfaces were then coated with metallic paint to provide a conducting surface. The beaten surface was made of aluminum, and therefore was already conductive. The statistics of the surfaces will be described below.

The first machined surface will be known as the Large Scale Surface (LSS) [5]. The surface grid is a single scale Gaussian surface. The standard height deviation of this surface is $3 \mathrm{~cm}$, and the correlation length is $8.6 \mathrm{~cm}$. The roughness scale of the surface is therefore considered large at frequencies from 4 to $12 \mathrm{GHz}$. This places the scattering behavior in the Kirchhoff region. Axline and Fung [6] quantified the mathematical requirements as

$$
\begin{aligned}
& \mathrm{k}_{1} \rho_{\mathrm{L}}>6 \\
& \rho_{\mathrm{L}}^{2}>2.76 \sigma \lambda
\end{aligned}
$$

where $\mathrm{k}_{1}$ is the wave number, $\rho_{L}$ is the surface correlation length, and $\sigma$ is the standard deviation of the surface heights. This large scale surface is painted with aluminum flake paint to 
make the surface conductive.

The second machined surface will be known as the Intermediate Scale Surface (ISS). This target is also a single scale Gaussian statistical surface, but with a height standard deviation of $0.25 \mathrm{~cm}$ and a correlation length of $2 \mathrm{~cm}$. At frequencies around $5 \mathrm{GHz}$, the surface satisfies the smallperturbation assumptions, and around $15 \mathrm{GHz}$ the surface falls in the high frequency or Kirchhoff region. This surface is painted with silver particle paint which was $66 \%$ silver. The sheet resistance rating for the paint is $0.01 \Omega / \mathrm{ft}$. An ohm meter was used to verify the high conductivity on the surface of the target. The conductivity of the aluminum paint on the previous target was not nearly as high.

The third surface is a multiscale surface with the standard height deviation of $0.16 \mathrm{~cm}$ and correlation length of $4.5 \mathrm{~cm}$. This surface is not a Gaussian surface, but the correlation function was matched with a sum of an exponential and a Gaussian term. The correlation function for this target is shown below.

$$
\rho(\tau)=0.5\left[\mathrm{e}^{-\tau / 0.29}+\mathrm{e}^{-(\tau / 4.5)^{2}}\right]
$$

The surface conductivity was assumed to be that of aluminum.

\section{Sustem and Data Reduction Description}

\section{Measurement System}

The measurements were made in the Bistatic Cross Section Range at the University of Texas at Arlington. The measurement hardware is a FM/CW swept system consisting of a Hewlett/Packard 8510 Network Analyzer, 8341A Sweeper, and a $8511 \mathrm{~A}$ Test Set. This type of measurement system has become a standard for many indoor and outdoor measurement systems.

\section{Data Acquisition}

The data was acquired over a bandwidth from 4 to 12 $\mathrm{GHz}$ for the Intermediate scale and hammer beaten targets. The large scale surface was measured from 8 to $12 \mathrm{GHz}$. No frequency behavior was observed from the large scale target due to the surface was close to the optical limit, and therefore would not show any frequency behavior over the frequency range of measurement system. The cross section data was calibrated using a six inch diameter sphere. For each incident angle 91 to 181 azimuth sweeps were acquired.

\section{Reduction Techniques}

The data sweeps were time-gated on a workstation with software which simulates the gating features on the HP 8510 . The cross section measurement sweeps were transferred to a set of workstations on a local network for reduction processing. A series of reduction programs were used to reduce the raw frequency sweeps into the scattering coefficient curves.

The first program used the large amount of azimuth data for each incident angle to compute the azimuth rotation correlation angle for each frequency to insure a sufficient number independent samples. The data sweeps were then averaged, keeping only those frequencies which had small correlation angle increments compared to the total azimuth aperture taken. This insured an adequate number of independent samples for a good estimate for the average cross section. The roughness of the surface was found to have an effect on the correlation angle. The large scale roughness target revealed the least amount of correlation in azimuth rotation. The hammer beaten surface was highly correlated due to the small roughness scale. The hammer beaten surface could be viewed as a flat conducting disk, which would be highly azimuth correlated, with a small roughness imposed on it.

The second program calculated the effective area of the target by integrating the normalized antenna pattern and the range effects over the circular surface target. The range effects were very small, usually in the third or four decimal place, but they were in included in the software for completeness. The scattering coefficient values were then computed by dividing the cross section values by the effective area of the target at each frequency. The frequency values remaining after the correlation test were then plotted to generate the frequency behavior of the surface for the specified polarization and incident angle.

\section{Results and Conclusions}

\section{Figure Discussion}

Figure-1 is a plot of the backscattering coefficient vs. incidence angle for the Large Scale Surface. The aluminum flake paint coating on this surface is not very conductive. The trend for the scattering curves follow the shape of the slope distribution as it should. Both the VV and the $\mathrm{HH}$ polarization curves have a similar response which is predicted by high frequency approximations.

Figure- 2 and Figure- 3 are plots of the backscattering coefficient vs. frequency for the Intermediate Scale Surface. The trends and levels of the experimental measurements match fairly well with the predictions of the theory. The peak in the curves shift toward the lower frequency direction as the angle of incidence increases.

Figure- 4 is a plot of the like polarized measurements of the Hammer Beaten Surface. The basic trends are similar, but the levels are very different. The correlation function in EQ(9) 
was fitted to very crude profile measurements, and therefore the actual correlation profile and standard deviation of surface heights may not be accurate. The surface profiles will be remeasured with a laser system in the future.

\section{Conclusions}

The computer generated surfaces provided rough surface targets with known statistical parameters. This made comparison with theoretical predictions much easier. The hammer beaten target was highly correlated with respect to azimuth rotation, and therefore the measurement set had less independent samples. The "ground truth" of the hammer beaten target was also in question. The theoretical model performed well in the comparisons shown, and other comparisons will be presented in the presentation of this work.

\section{References}

[1] A. K. Fung and G. W. Pan, "A scattering model for perfectly conducting random surfaces Part I. Model development", Int. Journal of Remote Sensing, Vol. 8, No. 11, pg. 1579-1593, 1987.

[2] M. F. Chen and K. S. Chen, "Numerical Verification of the Validity of the Improved Kirchhoff Model on Rough Surface Scattering", Proceedings of IGARSS 89 Symposium, Vancouver, Canada, pg. 1300-1303, July 1989.

[3] Z. Li and A. K. Fung, "Scattering from a Finitely Conducting Random Surface", PIERS Conference 1989, July 1989.

[4] J. D. Rochier, "The generation of surface targets with specified surface statistics", Int. Journal of Remote Sensing, Vol. 10, No. 7, pg. 1155-1174, 1989.

[5] C. E. Nance, A. J. Blanchard, and M. Chen, "Polarimetric Microwave Scattering from Known Randomly Rough Surfaces", Proceedings of IGARSS 88 Symposium, Edinburgh, Scotland, 1988.

[6] R. M. Axline and A. K. Fung, "Numerical computation of scattering from a perfectly conducting random surface", I.E.E.E. Transactions on Antennas and Propagation, Vol. 26, pg. $482-488,1978$.

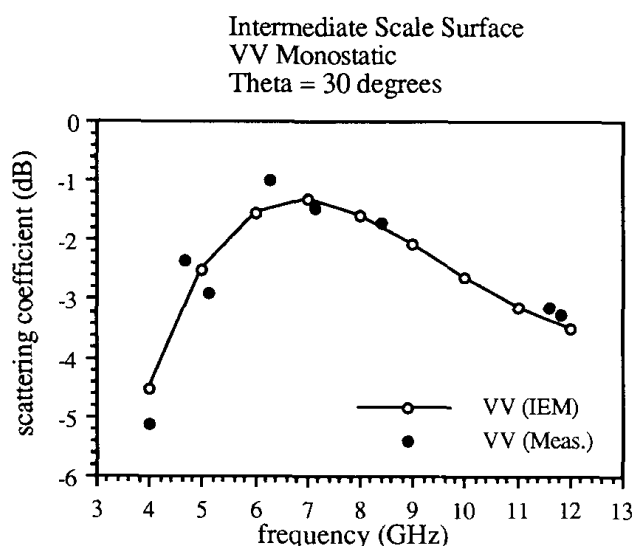

Figure-2: Backscatter from Intermediate Scale Gaussian Random Surface (Theta $=30$ degrees)

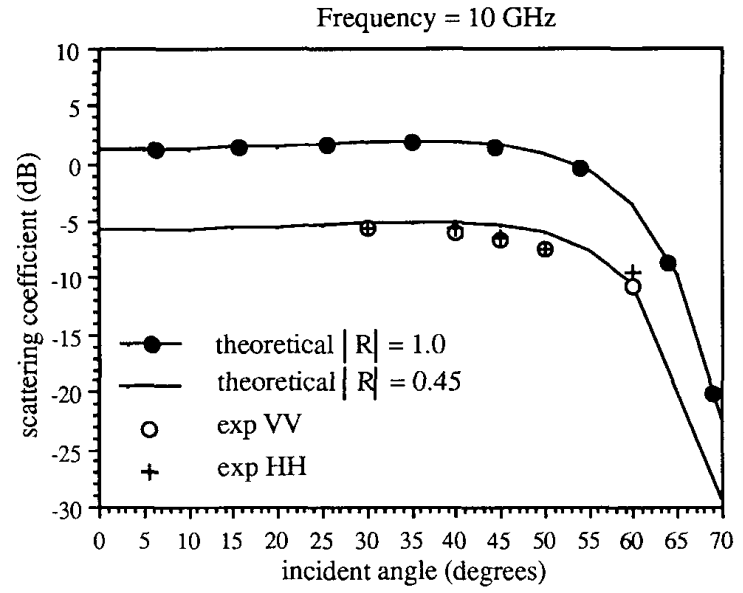

Figure-1: Backscatter from Large Scale Gaussian Random Surface (VV and HH polarization)

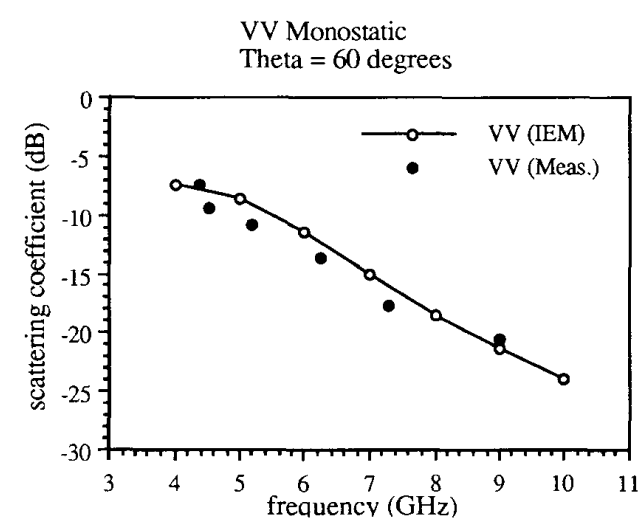

Figure-3: Backscatter from Intermediate Scale Gaussian Random Surface (Theta $=60$ degrees)

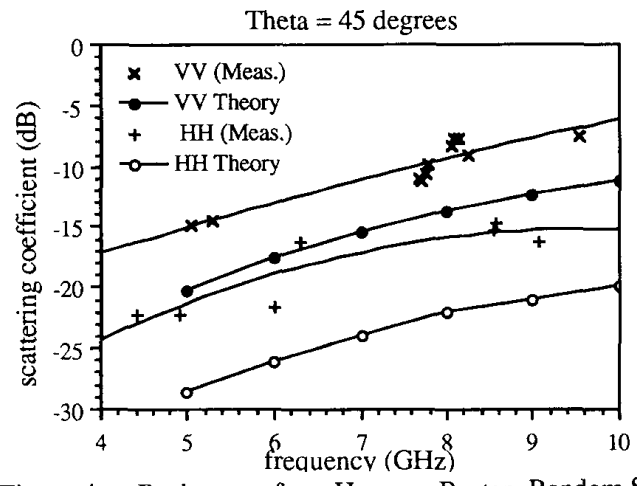

Figure-4: Backscatter from Hammer Beaten Random Surface (Theta $=45$ degrees) 